\title{
Numerical Investigation of the Plasma Formation in Distilled Water by Nd-YAG Laser Pulses of Different Duration
}

\author{
Laila H. Gaabour ${ }^{1}$, Yosr Ezz El Din Gamal ${ }^{1}$, G. Abdellatif ${ }^{2 *}$ \\ ${ }^{1}$ Department of Physics, Girl's Faculty of Science, King Abdul-Aziz University, Jeddah, KSA \\ ${ }^{2}$ Physics Department, Faculty of Science, Cairo University, Giza, Egypt \\ Email: Galila@sci.cu.edu.eg, galila@sci.cu.edu.eg
}

Received August 20, 2012; revised September 19, 2012; accepted September 26, 2012

\begin{abstract}
Water breakdown studies by Nd-YAG laser pulses of duration $100 \mathrm{fs}, 30 \mathrm{ps}$ and $6 \mathrm{~ns}$ at wavelength $1064 \mathrm{~nm}$ are preformed to investigate the physical mechanisms which couple the laser energy into the medium. Calculations are carried out applying a modified kinetic model of water breakdown previously developed by Kennedy (1995) to investigate the correlation between threshold intensity of breakdown and laser pulse length. The modifications considered the introduction of diffusion and recombination loss processes which might take place under the experimental conditions applied in these calculations. The validity of the model is tested by comparing the calculated threshold intensities and the experimentally measured ones where good agreement is shown. The study of the time evolution of the electron density clarifies the correlation between the pulse length and dominant ionization mechanism. The analysis of the spatial distribution of the electron density along the radial and axial distances of the focal spot showed that the size of the formed plasma increases with the increase of the pulse length. On the other hand, studies of self-focusing effect illustrated that under the investigated experimental conditions the effect of this process has an effective contribution only at laser pulses of the order of femtosecond scale when the laser beam is focused by a lens of a focal length $\geq 8.0 \mathrm{~cm}$. This result in turns assures that using femtosecond pulses in ophthalmic microsurgery could be a safe tool from the retinal damage.
\end{abstract}

Keywords: Laser Induced Breakdown; Water Breakdown; Electron Density; Self Focusing; Modeling of Plasma Formation; Laser Medicine

\section{Introduction}

Laser induced optical breakdown in aqueous media represents a challenging topic in applied physics. It received considerable attention for many years because it has various applications in medicine and biolog as well as it plays significant rules in the mechanism of plasma mediated ablation and photo-disruption. These represent the most important interactions between short laser pulses and tissue. Accordingly, it has great interest in medical applications especially in ophthalmic microsurgery and laser lithotripsy [1].

Although this phenomenon is extensively investigated both experimentally and theoretically [2-8], the mechanisms responsible for the threshold dependence on laser duration are still an open question. Experimental studies of this phenomenon revealed that, there are two main mechanisms that can lead to ionization and plasma formation namely; cascade ionization and multiphoton ionization $[9,10]$. Cascade or sometimes called avalanche
Ionization occurs with long pulse durations ( nanoseconds) and requires an initial quasi-free seed electron in the focal volume. This process tends to be impurity dependent and requires a quite condensed medium to allow many collisions within the long pulse duration. With ultra short pulse duration (picoseconds or femtoseconds) pure multiphoton ionization of the medium occurs where each molecule is independently ionized by the electric field, without the need of particle-particle interaction or seed electrons. This breakdown is thus independent of impurities in the medium and can be applied consistently to a variety of tissues. Moreover, the breakdown phenomenon is found to be more stable with the ultra short pulses while it is unstable with the nanosecond laser pulse scales [11]. Several theoretical models have been proposed to explain the physical phenomenon involved in the breakdown of a medium. These models describe the breakdown threshold intensity which determines the onsite of ionization and plasma formation considering 
these two mentioned mechanisms. Although these models could reasonably investigate the experimental measurements that carried out on gases and solids in aqueous media $[12,13]$, however, these models still did not give well understanding about the breakdown phenomenon.

Accordingly, in the present work a modified model previously developed by [4] based on the solution of a rate equation is proposed to investigate the plasma formation mechanism in distilled water induced by laser pulses of duration $100 \mathrm{fs}, 30 \mathrm{ps}$ and $6 \mathrm{~ns}$ at wavelength $1064 \mathrm{~nm}$ [14]. The model takes into account the generation of electrons due to the combined effect of both multiphoton ionization and cascade ionization processes. Moreover, loss processes due to electron diffusion and recombination are also considered in this analysis.

As a part of our study is the understanding and modeling the eye damage produced by exposure to laser pulses with different lenses. The aim of this study is to model the breakdown and plasma formation caused by different pulse duration in the area from the cornea to the retina. The result gained from this analysis is to find out the effect of self focusing which occurs during the interaction and propagation of the laser pulses. Measurements of the nonlinear effect and laser induced breakdown threshold for water indicate that water is a reasonable stimulant for the vitreous humor of the eye. In addition, water displays a nonlinear Kerr effect and self-focusing can occur at megawatt peak input powers. Indeed, these selffocusing leads to water breakdown which could explain the anomaly of the retinal damage caused by femtosecond pulses in the ophthalmic microsurgery [15]. Therefore, in this analysis we also present an investigation of the nonlinear effect associated with the propagation of laser radiation in a distilled water.

\section{Theoretical Formulations}

\subsection{Basic Equation}

In the present model the breakdown threshold is firstly considered to be independent of the laser beam diameter, i.e. the plasma formation is only time-dependent. Accordingly for a Gaussian laser pulse the temporal variation of the laser intensity is written as:

$$
I(t)=I(0) A\left(\mathrm{e}^{\frac{-(t-\tau)^{2}}{4 \tau^{2}}}-B\right)
$$

where $A(A=4.5208)$ and $B(B=0.7788)$ are constants, $I(0)$ is the laser peak intensity and $(2 \tau)$ is the laser pulse width (FWHM).

Secondly, in studying plasma propagation along the axial distance of the focal volume, the variation of the laser intensity is taken as a function of both space $z$ and time $t$ and is written as:

$$
I(t, z)=\frac{P_{\max }}{\pi w^{2}(z)} \exp \left[-4 \ln 2\left(\frac{t}{\tau}\right)^{2}\right]
$$

where $P_{\max }$ is the maximum laser power and $w(z)$ is the beam radius at any position $z$, which is given by

$$
w(z)=w_{0}\left(1+\frac{z^{2}}{z_{R}^{2}}\right)^{1 / 2}
$$

where $w_{0}$ is the beam waist and $z_{R}=n \pi w_{0}^{2} / \lambda$ is the Rayleigh length of the laser beam.

The focal volume is determined using the geometric optics consideration and has a cylindrical shape given by

$$
V=\frac{\pi d^{2}}{2}\left[(\sqrt{2}-1) f^{2} \theta / D_{0}\right]
$$

where $d$ is the beam spot size, $f$ is the focal length of the focusing lens, $\theta$ is the beam divergence and $D_{0}$ is the unfocused beam diameter. The breakdown criterion is taken to be the attainment of a critical electron density of $10^{19}-10^{20} \mathrm{~cm}^{-3}$ which agrees quite well with the experimental measurements given in [7].

Following to these parameters, the general form of the rate equation that describes the variation of electrons density under the combined effect of both multi-photon ionization and cascade ionization mechanisms in laser induced breakdown of water is written as:

$$
\frac{\mathrm{d} \rho}{\mathrm{d} t}=\left.\frac{\mathrm{d} \rho}{\mathrm{d} t}\right|_{m p a}+\eta_{c a s} \rho-D \rho-R \rho^{2}
$$

where $\rho$ is the free electron density, $\eta_{\text {cas }}, D$ and $R$ represents the ionization, diffusion and recombination rates respectively. The first two terms on the right hand side of this equation represent electron generation through multiphoton absorption $\left(\left.\frac{\mathrm{d} \rho}{\mathrm{d} t}\right|_{m p a}\right)$ and cascade ionization $\left(\eta_{\text {cas }} \rho\right)$. The remaining terms account for electron diffusion out of the focal volume $(-\mathrm{d} \rho)$ and the recombination loss $\left(-R \rho^{2}\right)$. For accurate computation in this model we take into account the depletion of water molecular due to ionization by subtracting the generated electron density from the molecular density. The water data required to solve Equation (5) is given in the following section.

\subsection{Water Data}

\subsubsection{Multiphoton Ionization Rate}

Following to [3] and other authors [4,14], in this model the water is treated as an amorphous semiconductor with a band gap energy (equivalent to the ionization energy $\Delta E=6.5 \mathrm{eV}$ ) [16]. To ionize a water molecule $K$ photons are required, where $K$ is the smallest integer greater than the ratio $\Delta E / h v$ and sometimes called the degree of non- 
linearity of multiphoton ionization, $h$ is Plank's constant and $v$ denoting the laser frequency. Consequently, the approximate expression for the multiphoton ionization rate in condensed media given by [17] is written as:

$$
\begin{aligned}
{\left[\frac{\mathrm{d} \rho}{\mathrm{d} t}\right]_{m p h}=} & \frac{2 \omega}{9 \pi}\left(\frac{m^{\prime} \omega}{\hbar}\right)^{3 / 2}\left[\frac{\mathrm{e}^{2}}{16 n c \varepsilon_{0} m^{\prime} \Delta E \omega^{2}} I\right]^{K} \\
& \exp (2 K I) \Phi\left(\sqrt{2\left(K-\frac{\Delta E}{\hbar \omega}\right)}\right)
\end{aligned}
$$

with

$$
\Phi(x)=\exp \left(-x^{2}\right) \int_{0}^{x} \exp \left(y^{2}\right) \mathrm{d} y
$$

In this equation $\omega$ is the angular frequency of laser light, $I$ represents the laser intensity,

$m^{\prime}=m_{e} m_{h} /\left(m_{e}+m_{h}\right)$ is the reduced exciton mass, $e$ is the electron charge, $n$ is the refractive index of water, $c$ is the velocity of light, $\varepsilon_{0}$ is permittivity of free space and $\Delta E$ is the ionization potential of water.

\subsubsection{Cascade Ionization Rate}

The assumed existing free electrons which are generated by multiphoton ionization can gain energy from the laser field through inverse Bremesstruhling absorption (IBA) in colliding with the water molecules. After several IBA events, the kinetic energy of the free electrons could exceed the ionization energy $\Delta E$. Then these electrons can collide and ionize another molecule creating new low energy free electrons. Following [4], the cascade ionization rate per electron is given by:

$$
\eta_{c a s}=\frac{1}{\omega^{2} \tau_{m}{ }^{2}+1}\left[\frac{\mathrm{e}^{2} \tau_{m}}{c n m \varepsilon_{0} \Delta E} I-\frac{m \omega^{2} \tau_{m}}{M}\right]
$$

where $\tau_{m}$ is the mean free time between electron-heavy particle collisions, $M$ is the mass of a medium molecule, (for water $M=3.0 \times 10^{-26} \mathrm{Kg}$ ), $m$ is the electron mass. The first term of this equation is related to the energy gain by electrons from the laser electric field where as the second term describes the energy transfer from electrons to water molecules during elastic collisions. For water the mean free time between momentum transfer collisions $\left(\tau_{m}\right)$ has not yet been measured, therefore we adopted the estimated value used in $[4,14]$ which is taken as $1 \mathrm{fs}$.

\subsubsection{Diffusion Loss}

The expression of diffusion rate per electron which describes the decrease of the electron density along the radial and axial distances of the cylindrical volume with radius $w_{0}$ and length $z_{R}$ is given by $[4,14]$ :

$$
D=\frac{\tau_{m} \Delta E}{3 m}\left[\left(\frac{2.4}{w_{0}}\right)^{2}+\left(\frac{1}{z_{R}}\right)^{2}\right]
$$

From this relation it is clear that the smaller the spot size the higher rate of diffusion loss. This in turn results in a higher breakdown threshold when the laser pulse exceeds the diffusion time. For pulse duration less than nanosecond diffusion time is greater than the laser pulse causing diffusion losses to be less probable.

\subsubsection{Recombination Rate Coefficient}

The recombination rate coefficient $\mathrm{R}$ is taken as the empirical value obtained by [2] through measurement of the decay plasma luminescence to be $2 \times 10^{-9} \mathrm{~cm}^{3} \cdot \mathrm{s}^{-1}$. For nanosecond pulses recombination losses during the laser pulse are quite important, while their effect might be negligible for shorter pulses.

\subsection{Method of Calculations}

Considering these rates, Equation (1) is solved numerically to obtain the threshold irradiance required to produce breakdown in water for a given pulse duration, using a Runge-Kutta fourth order technique with adaptive time step. Optical breakdown is assumed to occur when the free electron density obtained during the laser pulse exceeds the given critical value $\left(10^{19}-10^{20} \mathrm{~cm}^{-3}\right)$. The solution procedure for the plasma formation in water induced by laser pulses in each time step can be summarized as:

- The free electron density is obtained first at the center of the focal volume;

- The spatial distribution of the free electron density is identified as non-breakdown and breakdown regions. Then the corresponding plasma length is determined.

\subsection{Nonlinear Effect}

For completeness a study is carried out to investigate the effect of self-focusing on the breakdown of water under the experimental conditions considered in this analysis. This study has an important application in ophthalmologic microsurgery of a human eye. Accordingly, the dimensions of the focal spot are adjusted to fit the human eye configuration. The critical power for self focusing collapse in the absence of plasma generated is calculated using the formula [18].

$$
P_{c}=\frac{3.77 \lambda^{2}}{8 \pi n_{o} n_{2}}
$$

where $n_{2}$ is the nonlinear refractive index of water and is given by $n_{2}=3 / 2 B_{0} \lambda, B_{0}$ is the Kerr constant. $n_{2}$ is found to be $8.334 \times 10^{-16} \mathrm{~cm}^{2} / \mathrm{W}$ when the $B_{0}=4.7 \times 10^{7}$, at laser wavelength $1064 \mathrm{~nm}$ and $n_{0}$ is the linear refractive index (for water its value is 1.33) [19].

Computations are performed to determine the laser power required for breakdown as a function of both laser pulse length and focal length (input spot radius $w_{0}$ ). Its 
value is then compared with the obtained critical power value using the Equation (9).

\section{Results and Discussion}

Equation (1) is solved numerically under the experimenttal conditions given in [14] to investigate the breakdown of distilled water by Nd-YAG laser of wavelength 1064 $\mathrm{nm}$ at different pulse duration. Adopting the breakdown criterion which requires the attainment of an electron density of the order of $10^{19}-10^{20} \mathrm{~cm}^{-3}$ at the end of the laser pulse, a computer program is under taken to calculate the effect of: 1) laser pulse duration on the threshold intensity; 2) ionization mechanisms; 3) self focusing, as well as 4) the spatial distribution of the electron density along the radial and axial distances of the focal spot i.e. plasma propagation.

\subsection{Effect of Pulse Duration}

Computations are carried out to investigate the measurements of the breakdown threshold of distilled water as a function of pulse duration [14]. In doing so, Equation (5) was iteratively solved for different threshold intensities until the maximum electron density during the laser pulse equaled the critical electron density for optical breakdown. To examine the effect of loss processes on the threshold intensity, calculations are preformed in the presence and absence of each loss process. Figure 1 represents this relation where curve (1) refers to all processes, while curves (2) and (3) stand for the absence of recombination and diffusion respectively. For an easy comparison the experimentally measured values of [14] are also presented on the same figure (solid squares). It is clear from this figure that, there is a reasonable agreement between the calculated and measured threshold intensities $\left(\mathrm{I}_{\mathrm{th}}\right)$ over the whole range of pulse duration considered in this analysis when losses are taken into account (curve 1). Omission of recombination losses

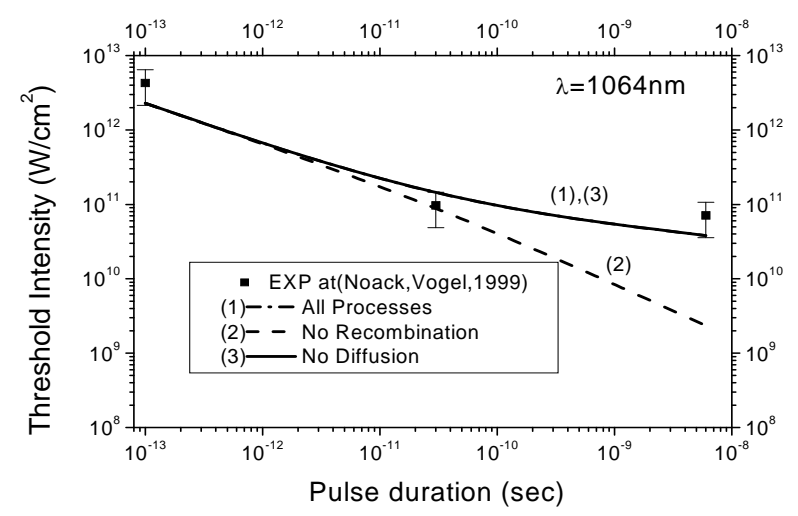

Figure 1. Comparison between calculated and measured threshold intensities as a function of pulse duration for distilled water. results in an observable deviation between the calculated and measured threshold intensity in particular at the longer laser pulse as shown by curve (2). Diffusion losses seems to have a negligible contribution to the threshold intensity since, its absence did not show any variation on the threshold intensity as illustrated by curve (3). This result revealed that over the laser pulse length examined experimentally, recombination losses have significant contribution only at $6 \mathrm{~ns}$ scale. The slow variation of the threshold intensity observed over the pulse duration range $\leq 500$ ps indicates that breakdown takes place mainly by multiphoton ionization process. While the pronounced dependence of the threshold intensities at pulse duration $\geq 500 \mathrm{ps}$, confirms the domination of collisional ionization process over the longer pulse duration.

\subsubsection{Effect of Ionization Mechanisms}

In the present work, studying the time evolution of the free electron density characterizes the interplay of multiphoton ionization in the presence of recombination losses during the breakdown process. Figure 2 represents this relation during the laser pulse under the combined contribution of multiphoton ionization and cascade ionization processes as well as the individual effect of multiphoton ionization calculated at the central point of the focal volume $\left(z_{0}, r_{0}\right)$. These are referred as curves (1) and (2) respectively in this figure for the laser pulses a) $6 \mathrm{~ns}$, b) $30 \mathrm{ps}$, and c) $100 \mathrm{fs}$.

From Figure 2(a) it is observed that the electron density starts to grow after a period of $0.2 \mathrm{~ns}$ with an electron density not exceeding $10 \mathrm{~cm}^{-3}$ as shown by curves (1) and (2). Beyond this time, curve (1) suffers a sudden increase reaching a value of $10^{20} \mathrm{~cm}^{-3}$ at a time of $0.3 \mathrm{~ns}$. Then its value remains almost constant up to $5.5 \mathrm{~ns}$ where it shows a slight decrease. While curve (2) (no cascade ionization) showed a gradual increase reaching to a value of $10^{7} \mathrm{~cm}^{-3}$ at the peak of the laser pulse, then it continue with this value up to its end. This behavior suggests that for nanosecond pulses at infra red wavelengths, the breakdown threshold in distilled water are determined by the intensity required to producing the first free electrons by multiphoton absorption.

At that intensity the rate of cascade ionization is so high that the breakdown proceeds almost instantaneously to the critical electron density $\left(10^{20} \mathrm{~cm}^{-3}\right)$. This explains the sudden increase of the electron density during the early stages of the laser pulse shown by curve (1) in this figure.

In Figure 2(b) (30 ps), however, the electron density during the early stages of the laser pulse showed similar behavior where it started with an electron density of one electron per $\mathrm{cm}^{-3}$ at about 2 ps for both curves (1) and (2), then curve (1) showed a gradual increase up to the end of the laser pulse, while curve (2) started with a slower in 


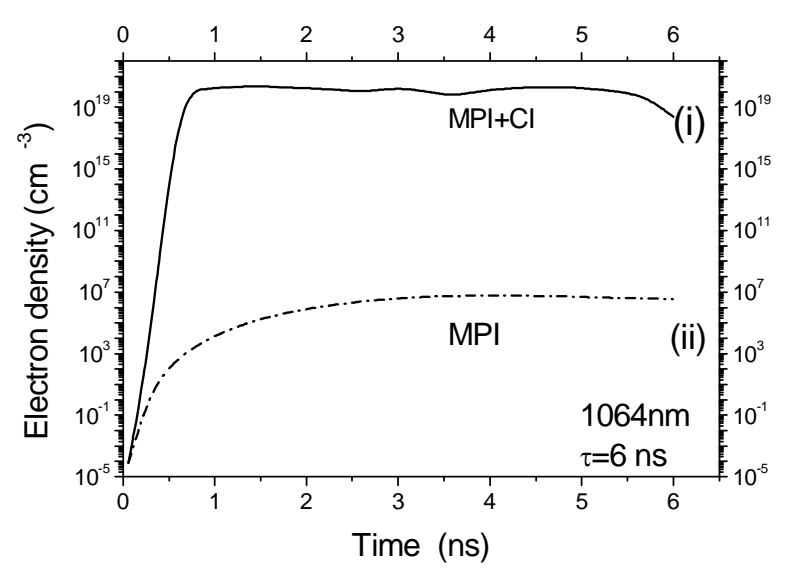

(a)

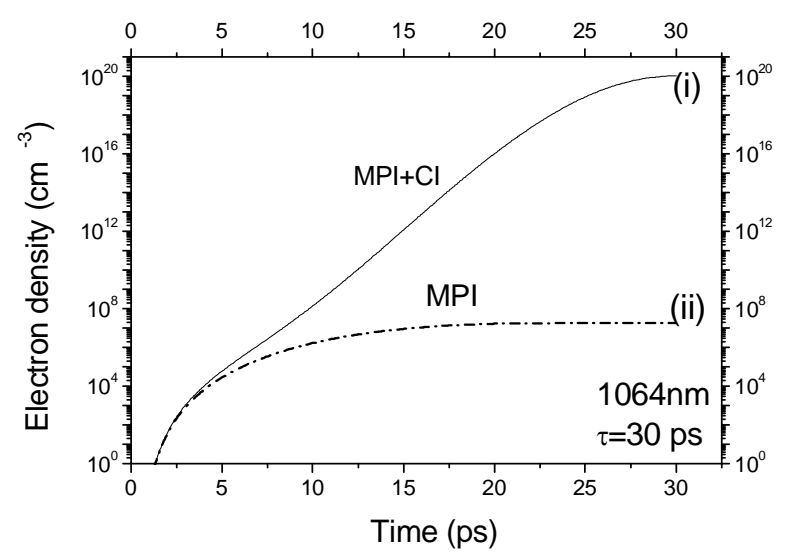

(b)

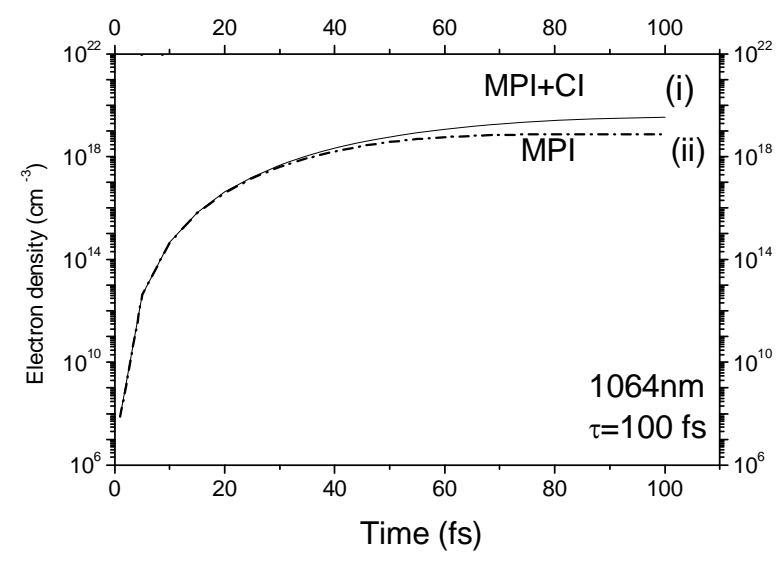

(c)

Figure 2. Evolution of free electron density, (i) combined effect of MPI and CI, (ii) MPI alone.

crease followed by an almost constant value at about $10^{7}$ $\mathrm{cm}^{-3}$ up to its end. From this figure it is clear that cascade-ionization contributes effectively to the breakdown of distilled water at this laser pulse length. This gradual increase of the electron density indicates the slow rate of cascade ionization during the first half of the laser pulse. This may be attributed to the competition between the two ionization mechanisms during this time which hinders the electron density growth. The increase shown by curve (1) during the second half of the laser pulse gives an evidence for the contribution of cascade ionization. On the other hand, in Figure 2(c) for the femtosecond laser pulse, curves (1) and (2) showed different behavior where they start with high values during the early stages of the laser pulse $\left(10^{8} \mathrm{~cm}^{-3}\right)$ followed by a constant value up to their end. The omission of cascade ionization (curve 2) showed only a slight decrease of the electron density during the second half of the laser pulse. This result supports the important role played by multiphoton ionization process to the breakdown of distilled water at the ultra short laser pulse (100 fs). This figure clarifies the important role played by the cascade ionization process in enhancing the electron density to reach the breakdown limits. Moreover, it showed also that for infra red wavelengths most free electrons are produced by cascade ionization even for the short pulse duration. Recombination losses occur near the end of the laser pulse (where a high electron density is achieved) as shown by the slow drop of the electron density illustrated by curve (1) Figure 2(a) for nanosecond pulses. Its influence becomes negligible for the ultra short pulses Figures 2(b) and (c) since its rate becomes slower compared to the laser pulse length.

\subsubsection{Spatial Distribution of the Electron Density along the Radial and Axial Distances of the Focal Spot: Plasma Length}

Taking the spatial distribution of the laser intensity as a Gaussian shape, calculations are preformed to obtain the distribution of the electron density in the cylindrical focal volume with axial distance $z_{R}$ and radial distance $w_{0}$. The aim of this analysis is to determine the actual size of the breakdown region (plasma length) as a function of the laser pulse length. The calculations are carried out at the threshold intensity for breakdown at three values along the axial distance namely; $z_{1}, z_{2}$ and $z_{3}$. At each value of $z$ the electron density is determined at three values along the radial axis of the focal volume $\left(r_{1}, r_{2}\right.$ and $\left.r_{3}\right)$ for the three laser pulses. Figures 3-5 represent this relation for $6 \mathrm{~ns}, 30 \mathrm{ps}$, and $100 \mathrm{fs}$ respectively. In these figures a, b, and c correspond to the values for the axial distances $z_{1}$, $z_{2}$ and $z_{3}$ at which the electrons density are calculated.

From Figure 3 (6 ns), it is clear that the evolution of electron density along the axial distance $\left(z_{1}, z_{2}\right.$ and $\left.z_{3}\right)$ takes the same trend during the laser pulse, where it shows a fast increase at the early stages followed by an almost constant value with a slight decrease at its end. Moreover, the values of the electron density at the end of the pulse undergo slow gradient along both the axial and radial distances. Table 1 shows these values together with the electron density calculated at the central focal 


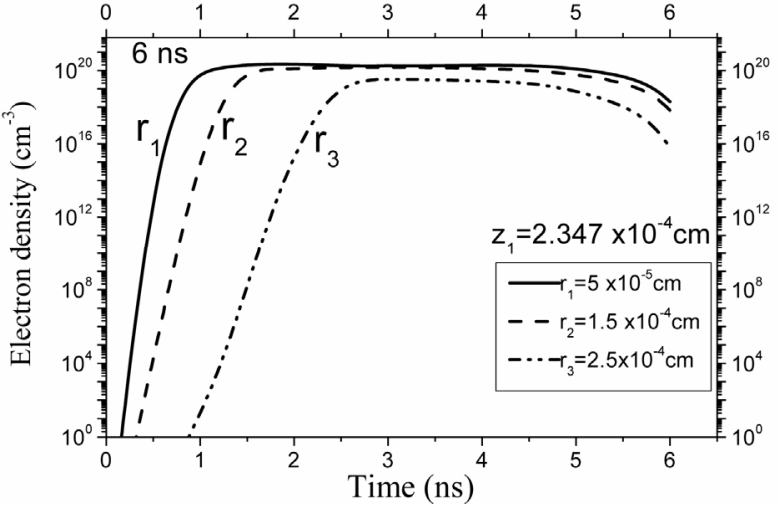

(a)

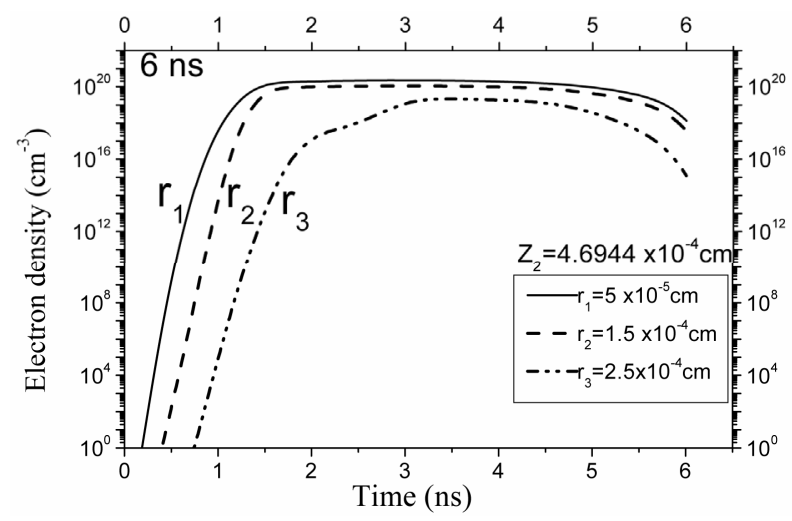

(b)

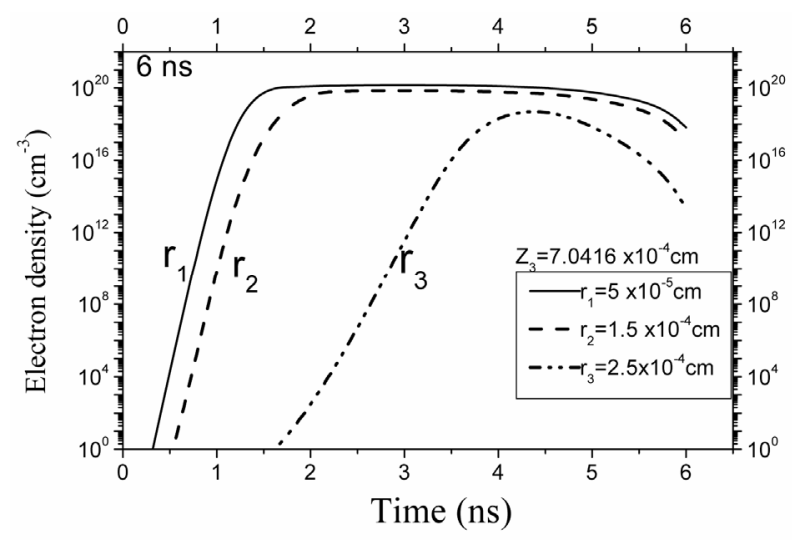

(c)

Figure 3. Spatial distribution of the electron density for laser pulse of $6 \mathrm{~ns}$ at three different locations along the axial distance (a) $z_{1} \mathrm{~cm}$, (b) $z_{2} \mathrm{~cm}$ and (c) $z_{3} \mathrm{~cm}$.

point $\left(z_{0}, r_{0}\right)$. This result reveals that the breakdown region almost cover the focal volume. At 30 ps pulse (Figure 4) different behavior is shown where the electron density along both the axial and radial distances showed a noticeable cut off resulting in a very small breakdown region just surrounding the center of the focal volume $\left(z_{0}\right.$, $\left.r_{0}\right)$. The calculated electron density along the axial distance (from $z_{0}$ to $z_{3}$ ) and radial values (from $r_{0}$ to $r_{3}$ ) are illustrated in Table 2. At the shorter pulse length (100 fs)
Table 1. Electron density at the end of the pulse along both the axial and radial distances $\left(z_{0}, r_{0}\right)$ at $6 \mathrm{~ns}$.

\begin{tabular}{ccccc}
\hline \multicolumn{5}{c}{ Electron density $\left(\mathrm{cm}^{-3}\right)$} \\
\hline $\mathrm{R}$ & $z_{0}$ & $z_{1}$ & $z_{2}$ & $z_{3}$ \\
\hline$r_{0}$ & $2.40 \times 10^{20}$ & $2.16 \times 10^{20}$ & $1.53 \times 10^{18}$ & $7.64 \times 10^{17}$ \\
$r_{1}$ & $2.16 \times 10^{20}$ & $1.93 \times 10^{20}$ & $1.35 \times 10^{18}$ & $6.50 \times 10^{17}$ \\
$r_{2}$ & $7.64 \times 10^{17}$ & $6.50 \times 10^{17}$ & $3.78 \times 10^{17}$ & $1.19 \times 10^{17}$ \\
$r_{3}$ & $1.00 \times 10^{16}$ & $6.24 \times 10^{15}$ & $1.18 \times 10^{15}$ & $2.34 \times 10^{13}$ \\
\hline
\end{tabular}

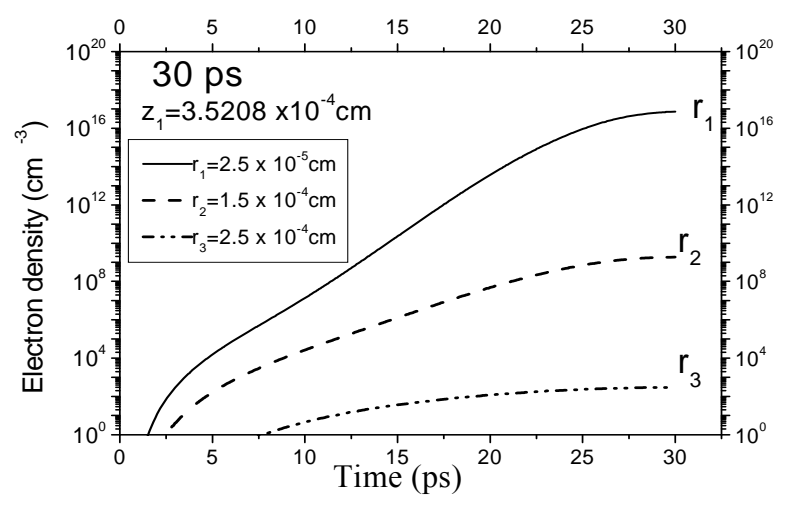

(a)

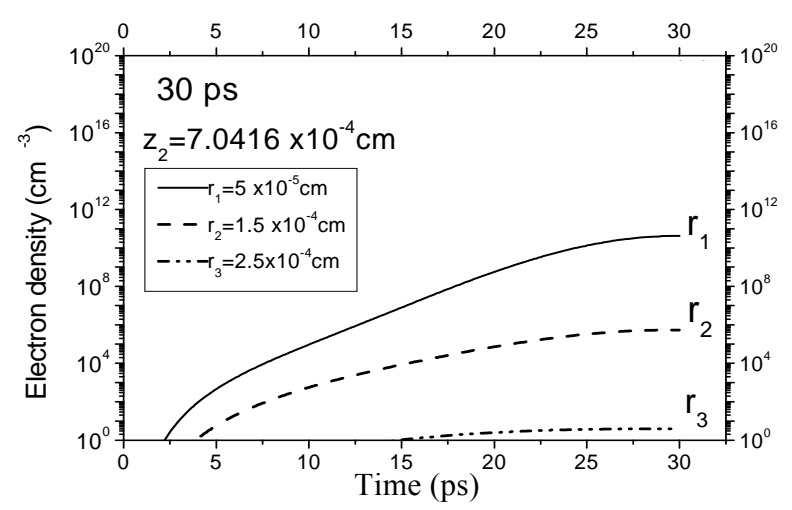

(b)

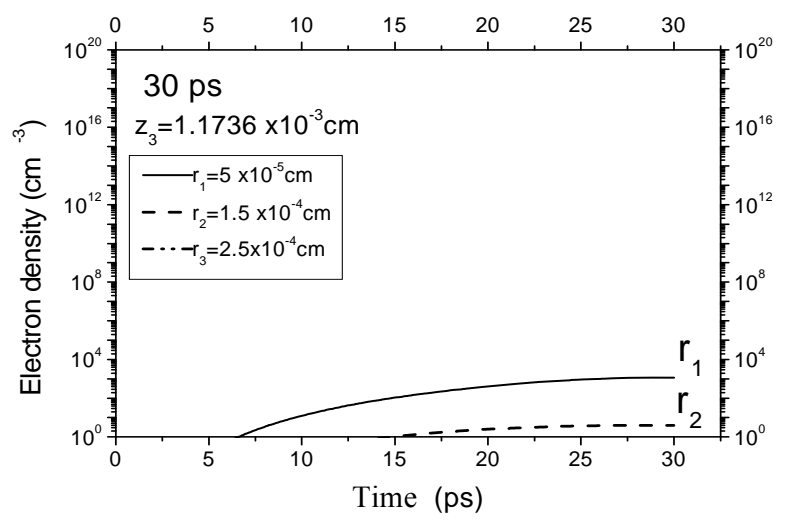

(c)

Figure 4. The same relation but for laser pulse of $30 \mathrm{ps}$. 
Figure 5 presents the time history of the electron density at the different locations along the axial and radial distances.

From this figure it is clear that the electron density is almost homogenously distributed in a volume surrounding the central region of the focal volume. The calculated electron densities at the different locations along the axial and radial distances are given in Table 3. Figures 3-5 depicted precisely the spatial distribution of the peak free electron density for the three laser pulses. It appears

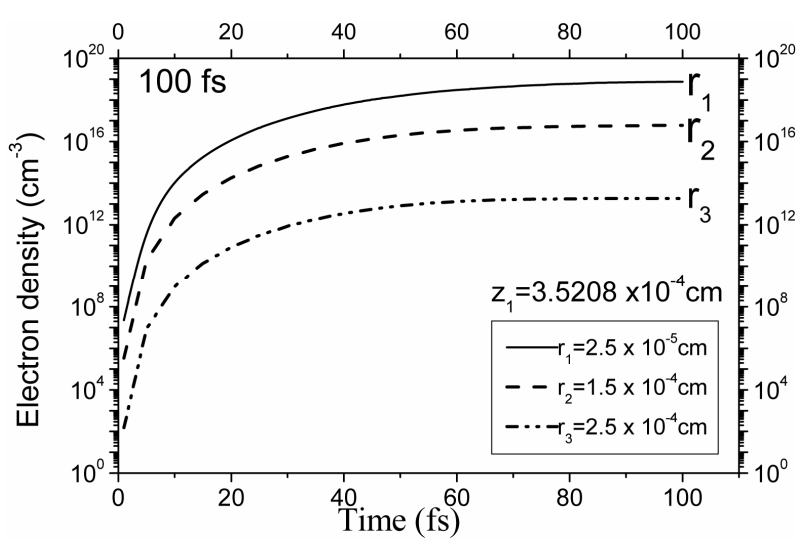

(a)

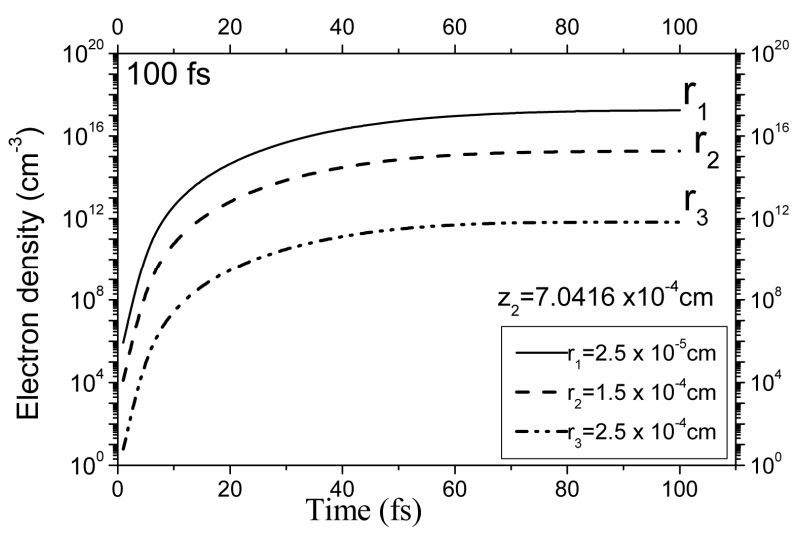

(b)

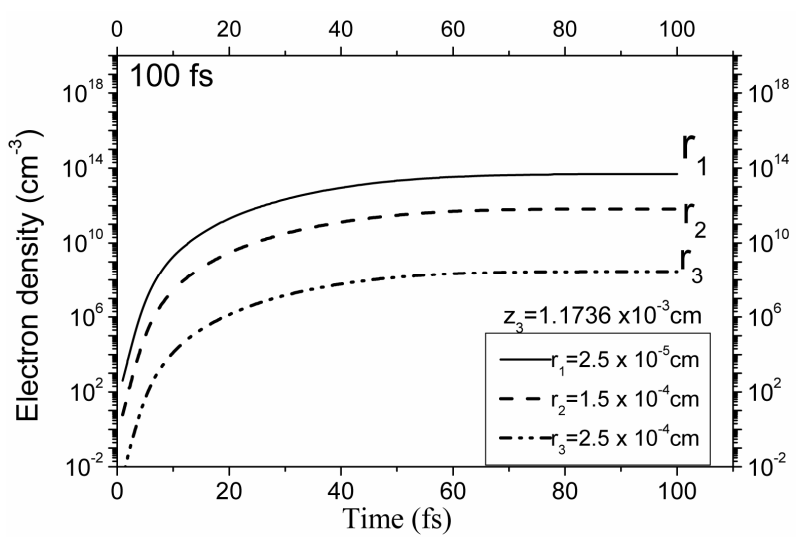

(c)

Figure 5. The same relation but for laser pulse of $100 \mathrm{fs}$.
Table 2. Electron density at the end of the pulse along both the axial and radial distances $\left(z_{0}, r_{0}\right)$ at 30 ps.

\begin{tabular}{ccccc}
\hline \multicolumn{5}{c}{ Electron density $\left(\mathrm{cm}^{-3}\right)$} \\
\hline$R$ & $z_{0}$ & $z_{1}$ & $z_{2}$ & $z_{3}$ \\
\hline$r_{0}$ & $1.13 \times 10^{20}$ & $1.41 \times 10^{17}$ & $6.52 \times 10^{10}$ & $1.42 \times 10^{3}$ \\
$r_{1}$ & $5.05 \times 10^{19}$ & $7.30 \times 10^{16}$ & $4.28 \times 10^{10}$ & $1.18 \times 10^{3}$ \\
$r_{2}$ & $6.52 \times 10^{10}$ & $1.87 \times 10^{9}$ & $5.47 \times 10^{5}$ & $3.94 \times 10^{0}$ \\
$r_{3}$ & $1.42 \times 10^{3}$ & $2.85 \times 10^{2}$ & $3.94 \times 10^{0}$ & $7.03 \times 10^{-4}$ \\
\hline
\end{tabular}

Table 3. Electron density at the end of the pulse along both the axial and radial distances $\left(z_{0}, r_{0}\right)$ at $100 \mathrm{fs}$.

\begin{tabular}{ccccc}
\hline \multicolumn{5}{c}{ Electron density $\left(\mathrm{cm}^{-3}\right)$} \\
\hline$R$ & $z_{0}$ & $z_{1}$ & $z_{2}$ & $z_{3}$ \\
\hline$r_{0}$ & $3.34 \times 10^{19}$ & $8.71 \times 10^{18}$ & $1.99 \times 10^{17}$ & $5.50 \times 10^{13}$ \\
$r_{1}$ & $2.87 \times 10^{19}$ & $7.52 \times 10^{18}$ & $1.74 \times 10^{17}$ & $4.86 \times 10^{13}$ \\
$r_{2}$ & $1.99 \times 10^{17}$ & $6.00 \times 10^{16}$ & $1.83 \times 10^{15}$ & $6.64 \times 10^{13}$ \\
$r_{3}$ & $5.50 \times 10^{13}$ & $1.81 \times 10^{13}$ & $6.64 \times 10^{11}$ & $2.87 \times 10^{8}$ \\
\hline
\end{tabular}

that the maximum value of the peak free electron density occurs at the focus point. At a glance the results shown in these figures can be used for predicting the maximum plasma length. Moreover, the obtained results may clarify that once optical breakdown occurs the focal volume will be divided into a breakdown and non-breakdown regions as shown in Figure 6. This figure represents the contour distribution of the electron density in the area surrounding the central focal point. Due to the symmetrical shape of the cylindrical focal volume, the contours are taken for just a quarter.

It is shown that at $6 \mathrm{~ns}$ the breakdown region extended beyond the central point along both axial and radial distances producing comparatively sizable plasma. For 30 ps, however, the breakdown region is confined to a very small size exactly at the central focal point surrounded with non breakdown region. At the shorter pulse length (100 fs) different behavior is shown where the formed plasma is elongated on the axial distance producing a pipe shape breakdown region surrounded by non breakdown zone.

\subsection{Effect of Self Focusing}

To test roughly the effect of self-focusing which is likely takes place during the interaction of high power laser with liquids, computation are carried out to investigate the experimental measurements given in [14]. In doing so, the threshold power is calculated in terms of the given spot size diameter $\left(2 w_{0}=5.0 \mu \mathrm{m}\right)$ and the calculated threshold intensity for the three laser pulses examined 


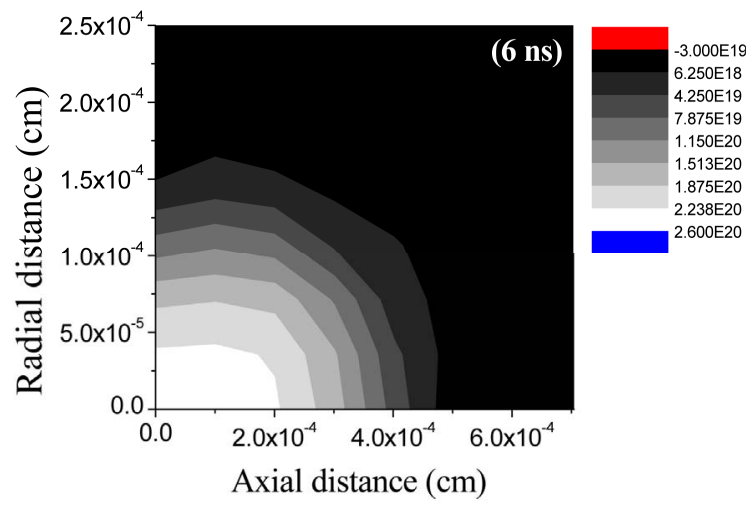

(a)

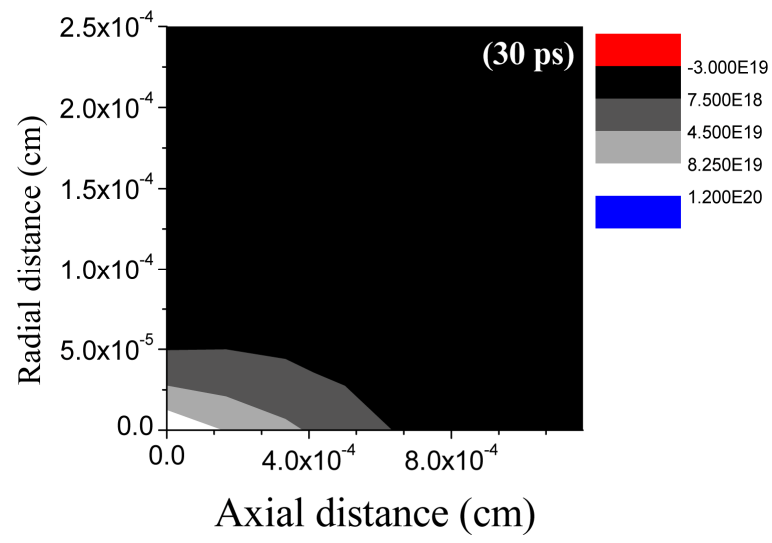

(b)

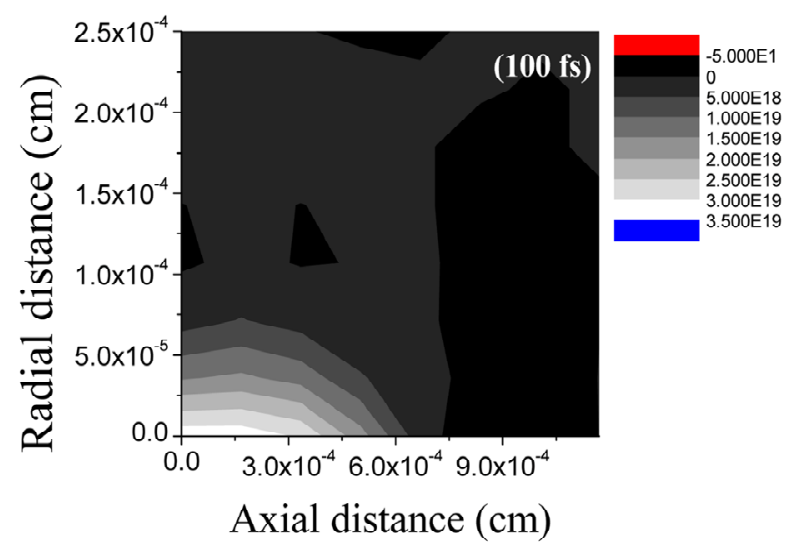

(c)

Figure 6. Contour representation for the electron density along the axial and radial distances of the focal volume for (a) $6 \mathrm{~ns}$; (b) $30 \mathrm{ps}$ and (c) $100 \mathrm{fs}$.

experimentally. Estimating the critical power for self focusing using Equation (9), it is found to be 1.53 MW.

Table 4 illustrates the calculated threshold power $\left(\mathrm{P}_{\text {th }}\right)$ at the three laser pulse lengths. From this table it is clear that these powers are much lower than the critical power for self focusing. Therefore, this phenomenon is unlikely to occur under the give experimental conditions. This might be attributed to the small spot size which corre-
Table 4. Threshold power versus pulse duration, for focused spot radius $2.5 \times 10^{-4} \mathrm{~cm}$.

\begin{tabular}{cccc}
\hline Laser pulse length & $100 \mathrm{fs}$ & $30 \mathrm{ps}$ & $6 \mathrm{~ns}$ \\
\hline Calculated threshold power (MW) & 0.44 & 0.03 & 0.007 \\
\hline
\end{tabular}

sponds to short focal length of the focusing lens $(\sim 4.0$ $\mathrm{cm})$.

An attempt is made to check the exact dependence of the focal length of the focusing lens on the threshold power for each laser pulse length. Knowing the beam divergence of the laser source $\left(\theta=1.148 \times 10^{-4} \mathrm{rad}\right.$.), it was possible to determine the threshold power corresponding to focusing lens with focal lengths vary between $1-10 \mathrm{~cm}$. Figure 7 presents a relation between the estimated values of the threshold power as a function of the focal length at 6 ns (curve 1), 30 ps (curve 2), and 100 fs (curve 3 ). Curves (1 and 2) showed very low values for the threshold power over the whole focal length range. For the ultra short laser pulse (curve 3) different behavior is shown, where the value of the threshold power showed a slight increase up to $5.0 \mathrm{~cm}$ followed by a faster increase reaching a high value exceeding the critical power for self focusing $(1.53 \mathrm{MW})$ at $10.0 \mathrm{~cm}$. In this study the critical power corresponds to a focal length of $8.0 \mathrm{~cm}$ as indicated in this figure. This result confirms the negligible contribution of the self focusing at the three laser pulses considered in this analysis when the laser beam is focused with a lens of focal length not exceeding $8.0 \mathrm{~cm}$.

\section{Conclusion}

In this study a modified numerical model based on a rate equation is applied to investigate the transit evolution of plasma in distilled water generated by focused short laser pulses. The study takes into account first, a Gaussian shape for the temporal distribution of the laser intensity and hence the electron density in the cylindrical focal volume. The rate equation is solved numerically using a fourth order Runge-Kutta method with adaptive time step control. The threshold intensities of the various laser pulses which are tested experimentally by Noack and Vogel [14] are computed and compared with the experimentally measured ones. Good agreement is obtained which confirms the validity of the modified model. Calculations are also preformed to simulate the start up and growth of the electron density. This was carried out for nano, pico and femto laser pulses. The results illustrated the effect of ionization mechanisms. It is also found that recombination losses act pronouncedly at the nanosecond domain. Secondly, the rate equation is solved numerically taking into account a Gaussian shape for the temporal and spatial distribution of the laser intensity in the 


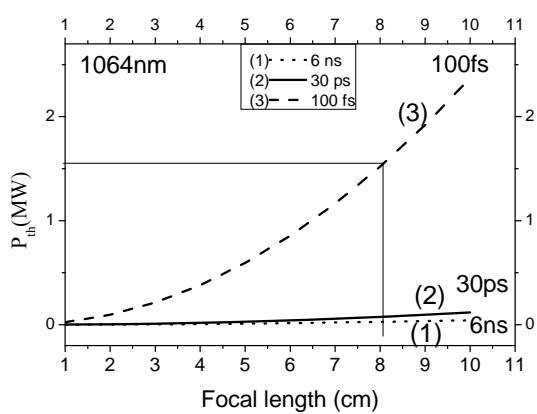

Figure 7. Threshold power versus focal length for (1) $6 \mathrm{~ns}$, (2) $30 \mathrm{ps}$, and (3) $100 \mathrm{fs}$.

focal volume. The beam waist is assumed to vary along the axial distance. The results of these calculations revealed deep understanding about the temporal evolution and spatial distribution of the free electron in the focal volume. The highest electron density is obtained at the centre focal point $\left(z_{0}, r_{0}\right)$. The maximum volume of the plasma is determined from the electron density distribution along the axial and radial distances and its value showed an increase with increasing the laser pulse. Moreover, the shape of the formed plasma varies with the laser pulse length. In addition, investigation of the effect of self focusing over the studied pulse length indicated that this process may play important role only for the femtosecond pulses when a focusing lens is used with focal length $\geq 8.0 \mathrm{~cm}$. No evidence of this effect is observed at $30 \mathrm{ps}$ and $6 \mathrm{~ns}$ pulse lengths. Accordingly, laser sources operating at the later pulse lengths as well as $100 \mathrm{fs}$ pulses focused with a lens of focal length less than $8.0 \mathrm{~cm}$ can be used safely for ophthalmic microsurgery.

\section{REFERENCES}

[1] A. Vogel, "Non-Linear Absorption: Intraocular Microsurgery and Laser Lithotripsy," Physics in Medicine and Biology, Vol. 42, 1997, p. 895. doi:10.1088/0031-9155/42/5/011

[2] F. Docchio, "Lifetimes of Plasmas Induced in Liquids and Ocular Media by Single Nd:YAG Laser Pulses of Different Duration," Europhysics Letters, Vol. 6, No. 5, 1988, p. 407.

[3] C. A. Sacchi, "Laser-Induced Electric Breakdown in Water," Journal of the Optical Society of America B, Vol. 8, No. 2, 1991, p. 337.

[4] P. K. Kennedy, "A First-Order Model for Computation of Laser-Induced Breakdown Thresholds in Ocular and Aqueous Media: Part I-Theory," IEEE Journal of Quantum Electron, Vol. 31, No. 12, 1995, p. 2241.

[5] Q. Feng, J. V. Moloney, A. C. Newell, E. M. Wright, K. Cook, P. K. kennedy, D. X. Hammer, B. A. Rockwell and C. R. Thompson, "Theory and Simulation on the Threshold of Water Breakdown Induced by Focused Ultrashort Laser Pulses," IEEE Journal of Quantum Electron, Vol. 33, No. 2, 1997, p. 127.
[6] A. Takita and Y. Hayasaki, "Dynamics of Femtosecond Laser-Induced Breakdown in Water," Proceedings of SPIE, Vol. 7201, 2009.

[7] J. Zhou, J. K. Chen and Y. Zhang, "Numerical Modeling of Transient Progression of Plasma Formation in Biological Tissues Induced by Short Laser Pulses," Applied Physics B: Lasers and Optics, Vol. 90, No. 1, 2008, pp. 141-148. doi:10.1007/s00340-007-2843-Z

[8] A. Sollier, L. Berthe and R. Fabbro, "Numerical Modeling of the Transmission of Breakdown Plasma Generated in Water during Laser Shock Processing," European Physical Journal Applied Physics, Vol. 16, 2001, pp. 131139.

[9] D. X. Hammer, R. J. Thomas, G. D. Noojin, B. A. Rockwell, P. K. Kennedy and W. P. Roach, "Experimental Investigation of Ultrashort Pulse Laser-Induced Breakdown Thresholds in Aqueous Media," IEEE Journal of Quantum Electronics, Vol. 32. No. 4, 1996, pp. 670-678. doi: $10.1109 / 3.488842$

[10] F. Docchio, P. Regondi, M. R. C. Capon and J. Mellerio, "Study of the Temporal and Spatial Dynamics of Plasmas Induced in Liquids by Nanosecond Nd:YAG Laser Pulses. 1: Analysis of the Plasma Starting Times," Applied Optics, Vol. 27, No. 17, 1988, pp. 3661-3668. doi:10.1364/AO.27.003661

[11] M. Bass and H. H. Barrett, "Avalanche Breakdown and the Probabilistic Nature of Laser-Induced Damage," IEEE Journal of Quantum Electron, Vol. 8, No. 3, 1972, pp. 338-343. doi:10.1109/JQE.1972.1076971

[12] C. Grey-Morgan, "Laser-Induced Breakdown of Gases," Reports on Progress in Physics, Vol. 38, No. 5, 1975, pp. 621-665. doi:10.1088/0034-4885/38/5/002

[13] C. B. Schaffer, N. Nishimura, E. N. Glezer and E. Mazur, "Ultrafast Laser-Induced Microexplosions: Explosive Dynamics and Submicrometer Structures," Proceedings of SPIE on Commercial Applications of Ultrafast Lasers, Vol. 3269, 1998, p. 36. doi:10.1117/12.312339

[14] J. Noack and A. Vogel, "Laser-Induced Plasma Formation in Water at Nanosecond to Femtosecond Time Scales: Calculation of Thresholds, Absorption Coefficients, and Energy Density," IEEE Journal of Quantum Electron, Vol. 35, No. 8, 1999, pp. 1156-1167. doi:10.1109/3.777215

[15] J. A. Powell, J. V. Moloney, A. C. Newell and R. A. Albanese, "Beam Collapse as an Explanation for Anomalous Ocular Damage," Journal of the Optical Society of America $B$, Vol. 10, No. 7, 1993, pp. 1230-1241. doi:10.1364/JOSAB. 10.001230

[16] F. Williams, S. P. Varma and S. Hillenius, "Liquid Water as a Lone-Pair Amorphous Semiconductor," Journal of Chemical Physics, Vol. 64, No. 4, 1976, p. 1549. doi: $10.1063 / 1.432377$

[17] L. V. Keldysh, "Ionization in the Field of a Strong Electromagnetic Wave," Soviet Physics JETP, Vol. 20, 1965, p. 1307.

[18] Y. R. Shen, "The Principle of Nonlinear Optics," Wiley, New York, 1984.

[19] E. U. Condon and H. O. Dishaw, "Handbook of Physics," 2nd Edition, Mac Grow Hill, New York, 1967. 